Didáctica. Lengua y literatura

ISSN: 1130-0531

http://dx.doi.org/10.5209/DIDA.57127

\title{
Propuesta para la enseñanza de la escritura a través de los géneros en la Educación Secundaria
}

\author{
Mari Mar Boillos Pereira ${ }^{1}$
}

Recibido: 27 de febrero de 2017 / Aceptado: 11 de mayo de 2017

Resumen. Las nuevas leyes de educación, así como las directrices metodológicas propuestas por los diferentes gobiernos autonómicos atestiguan una preocupación por el desarrollo de las habilidades lingüísticas de los estudiantes. Entre estas preocupaciones destaca el interés por el desarrollo de la capacidad escritora en la Educación Secundaria Obligatoria. Los estudios urgen generar intervenciones didácticas, como la de este artículo, en la que se aborde el trabajo de esta actividad de uso de la lengua desde un enfoque comunicativo y de manera transversal a todas las materias y no sólo aquellas del área de lengua. En este trabajo se analiza en detenimiento el caso del centro El Salvador-Maristas (Bilbao) en el que, conscientes de las carencias en enseñanza de la escritura, se ha generado un marco de trabajo para la enseñanza de esta habilidad en castellano en el primer curso de la ESO. Tras conocer las necesidades del centro y la documentación oficial, se presenta una propuesta didáctica acorde a las nuevas corrientes de estudio en materia de enseñanza de la escritura. Concretamente, se ha seleccionado el enfoque basado en los géneros discursivos en tanto que reúne las características necesarias para dar respuesta a las demandas diagnosticadas y por estar en armonía con los planteamientos oficiales. Esta propuesta pone de manifiesto la necesidad de revisar los modelos existentes para la enseñanza de las habilidades comunicativas y la necesidad, asimismo, de crear planes de intervención didáctica que se anticipen a los problemas que se han atestiguado en etapas educativas posteriores.

Palabras clave: competencia escritora; metodologías de enseñanza; profesorado de lenguas; enfoque basado en los géneros.

\section{[en] Proposal for Writing-teaching through Genres in Secondary Education}

Abstract. New educational laws, as well as the methodological guidelines proposed by the different regional governments attest a concern for the development of students' language skills. Among these concerns, emphasis must be placed on the interest for the development of the writing ability in Secondary school. Different studies urge to generate teaching interventions, such as the one in this article, to work on this skill from a communicative approach, and in a transversal way to all the subjects and not only to those linked to languages. This paper analyzes in detail the case of the El Salvador-Maristasschool (Bilbao). In this school, aware of the deficiencies in the teaching of writing, a framework has been generated for teaching this skill in Spanish in the first level of Secondary education. Once known the needs of the students and the official guiding principles, we present a didactic proposal based on the current studies on the teaching of writing. Specifically, we have selected the Genre-based approach as it meets the characteristics necessary to respond to the demands diagnosed and because it accomplishes official governmental guidelines. This proposal highlights the need to review existing models for the teaching of communicative skills and the need

\footnotetext{
Departamento de Didáctica de la Lengua y de la Literatura Universidad del País Vasco/Euskal Herriko Unibertsitatea mariadelmar.boillos@ehu.eus
} 
to create didactic intervention plans that anticipate the problems that have been witnessed in later educational stages.

Key words: writing skills; teaching methods; language teachers; genre-based approach.

\section{[fr] Proposition d'enseignement de l'écriture à travers les genres dans l'enseignement secondaire}

Résumé. La nouvelle législation de l'éducation, ainsi que les directives méthodologiques proposées par les différents gouvernements autonomes témoignent d'une préoccupation par le développement des compétences linguistiques des étudiants. Parmi ces préoccupations, il faut souligner l'intérêt du développement de la compétence en écriture dans l'enseignement secondaire obligatoire. Les plus récentes recherches invitent à générer des interventions didactiques dans ce domaine, comme celle qui va se présenter dans ce travail. L'intervention didactique proposée se centre dans le contexte de l'approche communicative de l'enseignement de la langue de manière globale, transversale à toutes les matières de l'école. Cet article présent l'étude de cas du centre scolaire Salvador-Maristas (Bilbao), qui en étant conscient des lacunes des élèves par rapport à l'écriture, a mis en place un cadre de travail pour le développement de l'enseignement-apprentissage de la langue castillane dans le premier cycle de l'enseignement secondaire obligatoire. Par conséquent, une fois détectés les besoins du Centre et connu la documentation officielle, une proposition didactique accordeaux nouvelles tendances de l'étudedans le domaine de l'enseignement de l'écriture a été présentée. De plus, l'approche fondée sur les genres discursifs qui met en évidence les variables nécessaires pour répondreaux demandes diagnostiquées et qui harmonise avec les énoncés officiels a été choisie. Cette proposition souligne le besoin de revoir les modèles existants pour l'enseignement de la compétence communicative et la nécessité, en outre, de créer des plans d'intervention éducative qui anticipent les problèmes témoignés dans les étapes ultérieures de l'éducation.

Motsclés:écriture, méthodes d'enseignement, professeurs de langues, approche fondée sur les genres discursifs.

Sumario: 1. Introducción. 2. Marco teórico. 2.1. La documentación oficial y la escritura en la ESO. 2.2. El enfoque basado en los géneros. 3. Marco empírico. 3.1. Objetivos, propuestas y necesidades del curso Lengua +1 . 4. Resultados y propuestas. 4.1. Aspectos generales de la propuesta. 4.2. Ficha técnica, competencias y objetivos didácticos. 4.3. Descripción de la actividad. 4.4. Evaluación. 5. Conclusiones. 6. Bibliografía.

Cómo citar: Boillos Pereira, M.M. (2017). Propuesta para la enseñanza de la escritura a través de los géneros en la Educación Secundaria. Didáctica. Lengua y literatura, 29, 2017, 11-28.

\section{Introducción}

El desarrollo de las habilidades lingüísticas y, concretamente, el de la escritura, ha recibido especial atención en las últimas décadas. El hecho de que se hayan revelado carencias a nivel universitario (Álvarez y Yañiz, 2015; Camps y Castelló, 2013) ha dejado patente una problemática que tiene su origen en etapas educativas precedentes. Desde las instituciones han destacado la importancia de trabajar desde un punto de vista comunicativo esta actividad de uso de la lengua, pero, además, se ha incidido en la relevancia de abordar el trabajo de la escritura de manera transversal a todas las asignaturas y no sólo a aquellas que están vinculadas a las lenguas (Ministerio de Educación y Ciencia, 2013). 
Así, en este trabajo se va a analizar en detenimiento el caso del centro El Salvador-Maristas (Bilbao) en el que, a partir de las carencias diagnosticadas, ya han diseñado un plan de trabajo piloto para la enseñanza de la escritura en $1^{\circ}$ de la ESO. El objetivo no es otro que, a partir del marco establecido por el centro y de acuerdo a las directrices oficiales, así como las corrientes contemporáneas de enseñanza de escritura, proponer un plan de trabajo que dé respuesta a las necesidades del estudiante y que medie ante las dificultades que se manifiestan en esta etapa educativa.

Con dicha finalidad, en primer lugar, se presentará el marco de estudio con una descripción pormenorizada de cuáles son las directrices a nivel institucional con respecto a la escritura en la ESO, concretamente $1^{\circ} \mathrm{ESO}$, y de qué propuestas existen en la actualidad para el tratamiento de la escritura de acuerdo a los últimos avances en la investigación en este ámbito. A continuación, se describirá el contexto educativo en el que se ha manifestado esta necesidad, se conocerán los mecanismos que se han puesto en marcha en este centro y, finalmente, se ofrecerá una descripción pormenorizada de la propuesta que vendría a cubrir el vacío diagnosticado.

\section{Marco teórico}

Un recorrido por la documentación oficial publicada por el Gobierno Vasco en materia de Educación deja en evidencia la importancia de generar nuevas maneras de enseñar en las que la lengua y, por extensión, la escritura adquiera una función destacada. A continuación, se presenta una síntesis de cuáles son las directrices oficiales a este respecto, concretamente en la Educación Secundaria, y se definen las características fundamentales del enfoque basado en los géneros como propuesta válida para dar respuesta a las demandas educativas actuales.

\subsection{La documentación oficial y la escritura en la ESO}

Entre las diversas publicaciones llevadas a cabo por el Gobierno Vasco en materia de educación parecen destacar tres documentos que son los que aquí se van a estudiar: el Currículo para la Educación Secundaria, las Orientaciones didácticas en materia de Lengua - Vasca y Castellana-y, por último, el programa Heziberri 2020.

En el primero de ellos, el Currículo (Gobierno Vasco, 2010), las lenguas, en este caso el euskera y el castellano, son definidas como herramientas de las que el estudiante tiene que apropiarse para ser capaz de comunicarse en las diferentes situaciones a las que puede verse expuesto, expresarse, administrar su conocimiento y pensar. Para ello, se establecen cinco retos que son desarrollar la comunicación oral, la escrita, la educación literaria, la capacidad metalingüística y, por último, la dimensión social de la lengua. Se trata de unos retos que, además, no funcionan de manera aislada, sino que se retroalimentan y que ayudan a alcanzar la competencia comunicativa (en adelante, CC) en toda su complejidad.

En este punto, y sin ánimo de profundizar en el tema, sí que parece reseñable apuntar a que el concepto de CC que se maneja en esta documentación tuvo su origen

A lo largo de este trabajo se abordará la documentación oficial en materia de educación con validez en el País

Vasco en tanto que es la CCAA en la que se encuentra el centro cuyo caso es objeto de estudio. 
en las teorías de Hymes (1971) quien apuntó, por primera vez, a este concepto como la capacidad para producir enunciados dentro de situaciones de comunicación en una lengua en concreto. En la actualidad, la definición de CC más extendida es aquella del Marco común europeo de referencia para las lenguas o MCER(Consejo de Europa, 2001) en el que se habla de ésta como una competencia que abarca saberes de tipo lingüístico, sociolingüístico y pragmático.

En cuanto al segundo reto, el de la escritura, además de presentar la necesidad de que el estudiante pueda desenvolverse en diferentes ámbitos de uso, también apunta a que la escritura resulta fundamental para el desarrollo de las "habilidades cognitivo lingüísticas que inciden en la construcción del conocimiento en todas las áreas" (Gobierno Vasco, 2010, p. 4). Se destaca, así, el valor epistémico de la escritura (Serrano, 2014) y se subraya la relevancia que tiene aprender a escribir para la comprensión y aplicación de los conocimientos de las diferentes materias.

Por lo que respecta al primer curso de la ESO, nivel en que se centra este estudio, se observa que la escritura y la lectura se abordan de manera íntimamente relacionada. Se espera que el estudiante comprenda, interprete y produzca textos propios de diferentes medios (sociales, académicos...) en los que haya una identificación del tema, objetivos, ideas principales, así como de sus características específicas (registro, canal...). Estos textos, además, pueden utilizar como canal tanto medios físicos, como digitales o audiovisuales; y sirven como herramienta para el trabajo de valores.

Destaca el hecho de que los textos en este documento se definan en relación a su tipología. A consecuencia de ello, los estudiantes tienen que ser capaces de comprender, interpretar y producir textos de naturaleza expositiva, narrativa, descriptiva, etc. Se espera que el estudiante identifique la identidad comunicativa de los escritos para dar respuesta a la tarea, previa planificación de la misma, con mayor efectividad. Es decir, de acuerdo a unos parámetros de un nivel aceptable de adecuación, coherencia, cohesión y corrección. No obstante, para lograr ese fin, además de haber desarrollado la capacidad de planificación, es necesario también hacer un uso estratégico de los conocimientos y los procesos cognitivos asociados a la textualización y a la revisión. De la misma manera, se espera un dominio razonable de las diferentes herramientas (diccionarios, bibliotecas, nuevas tecnologías...) que puedan contribuir a la mejora de su producción textual. Consecuentemente, los criterios de evaluación están directamente relacionados con estas premisas que aquí se han presentado.

Otro de los documentos de relevancia es el de Orientaciones didácticas para las materias de Lengua Castellana y Lengua Vasca (Gobierno Vasco, 2011). Se trata de un documento en el que este órgano pone de manifiesto su intención de incorporar el enfoque de las competencias básicas a la práctica docente. Se recupera el concepto de lengua presentado en el Currículo, el concepto también de escritura que se ha presentado y se destaca la importancia a las lenguas en relación a las diferentes competencias como son aprender a aprender, autonomía e iniciativa personal, la asociada a las matemáticas, cultura científica, tecnológica y de la salud; tratamiento de la información y competencia digital; cultura humanística y artística y social y ciudadana.

La enseñanza de las lenguas, así como de la escritura, se entiende desde un enfoque comunicativo y se destaca la importancia de diseñar herramientas de aprendizaje en las que se tengan en cuenta los roles, la evaluación, los contenidos y el tiempo con el fin de que se produzca una adecuada interiorización de los conocimientos. Se ofrecen, además, distintas pautas metodológicas entre las que se encuentra el trabajo de la escritura desde tipologías comunes para facilitar el tratamiento integrado de las 
lenguas y la transferencia de los conocimientos. En oposición, al hablar de la idea de introducir las tareas como metodología de enseñanza, se sugiere abordar la escritura desde los géneros para poder conocer la finalidad, los destinatarios, la estructura, etc. (Camps, 2003).

De la misma manera, cabe detenerse en las directrices que el Gobierno Vasco establece en el documento Heziberri 2020 (Gobierno Vasco, 2014) cuya finalidad es establecer un modelo educativo pedagógico que difiere significativamente del planteado en las leyes orgánicas como son la LOE o la LOMCE. En este documento, fruto de una herencia socioconstructivista, se defiende la enseñanza de las lenguas desde un enfoque social, interactivo y constructivista - como herramienta de aprendizaje - en el que el dominio de la lengua es sinónimo de éxito en las diferentes situaciones de comunicación, así como de un mejor desarrollo para el resto de competencias básicas para la formación integral de los estudiantes. Todo ello en un currículo en el que haya un tratamiento integrado de las lenguas que favorezca la formación bilingüe o trilingüe, según el caso.

En relación a las sugerencias didácticas que aporta este documento, cabe indicar que se alude a la necesidad de conocer y seleccionar los ámbitos y situaciones de comunicación reales a las que va a verse expuesto el estudiante para conocer sus necesidades y, así, hacer un diseño apropiado de los materiales. Se trata, en definitiva, de generar un aprendizaje significativo dentro de dimensiones que van desde lo personal, a lo social y lo académico-laboral.

Por último, cabe mencionar que, a pesar de que en este trabajo sólo se hayan mencionado directrices propuestas desde el Gobierno Vasco, todos estos planteamientosson fruto de un movimiento supraestatal impulsado por la Comisión Europea. Desde las instituciones europeas se busca dar un giro hacia lo comunicativo en la educación lingüística; un giro que conlleva una atenuación de los contrastes entre las enseñanzas de lenguas maternas y lenguas extranjeras. En definitiva, no se puede afirmar que el foco en las competencias clave sea un planteamiento que se esté produciendo únicamente en esta realidad, sino que se trata de un cambio de miras que afecta a toda la comunidad europea (Eurydice, 2002).

\subsection{El enfoque basado en los géneros}

Los géneros, ya desde Bajtin, precursor de este concepto (1979), son entendidos como formas reconocidas por los miembros de una comunidad de habla con naturaleza estable. Se trata de entidades de tipo social que surgen de las necesidades comunicativas de los hablantes que participan en el acto comunicativo, así como de los contextos en los que tienen lugar. Son, además, estructuras dinámicas (Raimes, 1998) que afectan en nuestras maneras de actuación dentro de nuestra cultura (Martin, 2001).

Los géneros se diferencian de las tipologías en tanto que, según Swales (1996), todos los textos que conforman un mismo género comparten el mismo objetivo y son identificados por sus contenidos, estructuras o estilo por todos los hablantes de esa comunidad (Parodi, 2008). Estas formas surgen del análisis del contexto y de las diferentes demandas lingüístico-estructurales propias de cada situación de comunicación (Hyland, 2003). Situaciones que, además, siempre están sujetas a las características del acto dialógico en el que se producen y, por tanto, tienen que tener 
en cuenta la audiencia, el intercambio comunicativo previo y la intervención de otras posibles voces.

Frente a estos, las tipologías, estáticas desde el punto de vista diacrónico y sincrónico, se clasifican en relación a características semántico-sintácticas que dan lugar a cinco formas estables: descripción, narración, exposición, argumentación y dirección (Werlich, 1975). Esta clasificación es la más empleada y recurrente en el ámbito de la didáctica de lenguas (Alexopoulou, 2010).

El enfoque basado en los géneros toma como punto de partida el género y lo sitúa como eje de un sistema de enseñanza de la escritura. Si ser competente desde el punto de vista comunicativo supone dominar los niveles lingüístico, sociocultural y discursivo-pragmático (Instituto Cervantes, 2016), el género se presenta como una herramienta válida para la interiorización y adquisición de todos los conocimientos que derivan de ellos (Zayas, 2012). El género "está basado no solo en las características estructurales, sino también en aspectos contextuales y cognitivos relevantes" (Arias, 2013, 40), a diferencia de los sistemas de enseñanza tradicionales que estaban centrados en lo lingüístico y textual (Hernández y Quintero, 2001). Así, en este nuevo enfoque, se propone una enseñanza fundamentada en la idea de literacidad, en la idea de potenciar los aspectos sociales, contextuales y facilitar su necesidad de comunicarse (Arias, 2013; Hyland, 2007). "Genre theory seeks to (i) understand the ways individuals use language to orient to and interpret particular commnicative situations, and (ii) employ this knowledge for literacy education" (Hyland, 2003, 22).

El procedimiento que promulga esta metodología es el siguiente. En primer lugar, se llevaría a cabo un análisis de las necesidades comunicativas del estudiante. Gracias a ello se podrán establecer los objetivos y los contextos de trabajo y, por extensión, la selección de los géneros y de los conocimientos sociocognitivos y lingüísticos necesarios para llevar a cabo dichos géneros. Estos deberán seleccionarse de mayor a menor grado de familiaridad y complejidad. Por último, tendrá lugar el diseño de la tarea que se basará en los objetivos de aprendizaje y los contenidos propios del nivel educativo del estudiante (Hyland, 2007).

Este autor habla también de los diferentes beneficios de este enfoque. Entre ellos destacan el carácter explícito de esta metodología, la sistematicidad, la relación con las necesidades, el empoderamiento, el despertar de la capacidad crítica y las herramientas que proporciona (Hyland, 2003); a los que añaden Zayas y Pérez Esteve (2016) la posibilidad de trabajar de manera interdisciplinar y la posibilidad de elicitar los conocimientos previos de los estudiantes. Otro de los puntos fuertes de este enfoque es que promueve tanto la toma de conciencia individual, como colectiva (Moyano, 2005). Así, se favorece el descubrimiento gradual de los conocimientos y el trabajo colaborativo. El docente, en ese marco de trabajo, tiene la posibilidad de afincar su rol de guía, de negociador (Hyland, 2003) y llevar a cabo tareas para establecer el contexto, los modelos, generar situaciones de noticing o de reconocimiento, favorecer la construcción guiada de los textos, la monitorización independiente de los textos y el establecimiento de redes para la inclusión de conocimientos nuevos (Feez, 1998).

Por último, la evaluación adquiere un valor capital. El seguimiento llevado a cabo por el docente de todo el proceso de composición del texto lo capacita para tomar decisiones con respecto a cómo abordar las diferentes etapas de la enseñanza y posibilita la intervención de tipo formativa durante el proceso. No se trata de calificar, sino de emplear la evaluación como herramienta para sustentar el aprendizaje y para revisar las debilidades que no están permitiendo al estudiante llevar a cabo 
con éxito la tarea comunicativa (Hyland, 2004). Este hecho ayuda significativamente en el acercamiento docente-estudiante, así como en la mejora del autoconcepto, la autocrítica y la motivación de este último (Hyland, 2007).

Sin embargo, pese a todos los beneficios mencionados y los artículos que apuntan a la importancia de este enfoque, apenas existen propuestas para el trabajo a través de los géneros en la Educación Secundaria. De hecho, se apunta a que aún predominan los materiales en los que la escritura no se trabaja desde la comunicación (Zayas, 2012). Además, son materiales en los que los textos que funcionan como input están creados ad hoc y en los que la tarea de escritura sigue siendo subsidiaria a las tipologías textuales (González-Caravaca, 2014).

\section{Marco empírico}

El centro escolar sobre el que se construye esta propuesta didáctica es el centro El Salvador-Maristas, ubicado en el Casco Viejo de Bilbao. Se trata de un centro educativo con una larga trayectoria en la ciudad — su fundación se remonta al 1918 — y en el que, desde una misión y unos valores de la comunidad Marista se imparte una formación educativa en un entorno religioso a niños y jóvenes. En él trabajan, en la actualidad, los ocho miembros que conforman el órgano directivo, 85 docentes y personal de administración y servicios.

En el Plan estratégico vigente (PE 2013-2016) del centro se aboga por establecer una serie de líneas de actuación sobre los que construir la formación de los estudiantes. Entre ellas se encuentra el rol evangelizador, la búsqueda del bienestar de la comunidad educativa, la formación continua de los docentes, la inclusión de las familias en la vida del centro y, por último, la sostenibilidad. Estas ideas se ven materializadas en los diferentes documentos que forman parte del Plan Educativo del Centro.

Sin ánimo de indagar en todos ellos, sí que parece de interés conocer las ideas presentes en el Plan de plurilingüismo y el Proyecto curricular de las materias de Lengua Castellana y Vasca y Literatura. El primero de ellos resulta de interés por su peso en el objetivo de adaptarse a las demandas lingüísticas de la realidad contemporánea. Acorde a las directrices de los órganos europeos, se aboga por formar a ciudadanos competentes en diversas lenguas que sean capaces de valorar la riqueza que subyace a cada lengua y, por extensión, a cada cultura. Es objetivo de este centro, por tanto, contar con profesionales capaces de dominar diferentes lenguas y que estén al tanto de las diferentes corrientes de enseñanza que se están desarrollando para favorecer la construcción de una Europa plurilingüe y de unos hablantes competentes en las lenguas que se estudien.

En lo que respecta al Proyecto curricular de las materias Lengua Castellana y Literatura y Lengua Vasca y literatura, un primer acercamiento al documento lleva a comprobar la estrecha relación de éste con las premisas del plan previamente citado. $\mathrm{Y}$ es que los diferentes objetivos que se señalan giran en torno al desarrollo de la CC del estudiante. Ese desarrollo será acorde a la etapa educativa en el que se encuentre el estudiante y acorde a las competencias básicas.

En este documento, se presentan, además, 11 retos que (ver Tabla 1), adaptados al nivel de los estudiantes, determinarán los contenidos, la metodología y la evaluación en cada curso. 
La comprensión de discursos orales y escritos de diversos ámbitos de uso de la lengua

La expresión e interacción oral y escrita

La habilidad de conocer e interpretar el entorno a partir de los conocimientos sociolingüísticos

El reconocer el plurilingüismo y las particularidades que derivan de él

La reflexión en torno a las estrategias comunicativas

Reconocer los mecanismos de adecuación, coherencia y cohesión de las diferentes lenguas y en las diferentes actividades de uso de la lengua

Despertar el espíritu crítico y la capacidad para el tratamiento y procesamiento de la información

La reflexión acerca de los propios procesos de aprendizaje y las estrategias para la transmisión de los conocimientos

El conocimiento de las convenciones propias de los diferentes géneros literarios en su dimensión diacrónica y sincrónica

El valorar el patrimonio literario

El disfrute mediante el producto literario

Tabla 1: Retos establecidos por el centro para las materias Lengua Castellana y Lengua Vasca (Fuente: Proyecto curricular de las materias Lengua Castellana/Vasca y Literatura. Información proporcionada por el centro)

Se busca, además, una metodología participativa, que motive a los estudiantes, que favorezca el aprendizaje autónomo y colaborativo, que recurra al uso de las nuevas tecnologías, que busque la funcionalidad y la adaptabilidad en horarios y espacio $\mathrm{y}$, por último, una metodología que tome medidas para asegurar una atención a la diversidad y a las necesidades específicas de los estudiantes.

De lo arriba mencionado se desprende la importancia otorgada a la formación en $\mathrm{CC}$, pero este interés se pone más de manifiesto, si cabe, al analizar la oferta educativa del centro para el primer ciclo de la ESO. Los programas nos permiten observar que en el primer curso se oferta una subasignatura denominada Lengua +1 en la que las enseñanzas giran en torno a la formación en dos actividades de uso de la lengua: la escritura y la lectura. Así también, en segundo curso se oferta la asignatura denominada Taller de comunicación, tanto en euskera como en castellano, en la que se trabaja la $\mathrm{CC}$ a diferentes niveles.

Este trabajo se centra en la primera de las subasignaturas citadas. Por ello, en el siguiente apartado se matizarán las características de esa asignatura de Lengua Castellana y Literatura, el porqué de esa fragmentación en la modalidad +1 y cuáles son los objetivos y las competencias que se trabajan en esta última. 


\subsection{Objetivos, propuestas y necesidades del curso Lengua +1}

El programa de la asignatura de Lengua Castellana y Literatura se construye sobre cinco bloques de contenidos que dan respuesta a las directrices de la documentación oficial:

a. Sobre la lengua, la literatura y la dimensión social: aspectos relativos a los niveles morfológico, sintáctico, léxico-semántico...; aspectos literarios, ...

b. Expresión oral

c. Expresión escrita

d. Comprensión escrita

e. Comprensión oral

En cuanto a la expresión escrita, el programa de la asignatura presta especial atención a cuáles son los géneros, las normas ortográficas... así como a cuáles son los criterios de evaluación, indicadores y técnicas que derivan de los bloques previamente mencionados. Estos criterios inciden en la importancia de comprender e interpretar el significado de textos producidos en diferentes contextos comunicativos y ser capaces de identificar tanto los elementos de la situación en la que se producen, como los rasgos textuales, discursivos e intencionales a los que recurre el autor. Así también, se destaca la necesidad de ser capaces de producir textos propios de distintas tipologías textuales (narración, exposición, descripción) y de distintos ámbitos de uso de acuerdo a las características propias de la situación comunicativa en la que se producen y haciendo un uso adecuado de los principios de coherencia, cohesión, corrección y adecuación. No obstante, y de acuerdo a los problemas diagnosticados en etapas educativas posteriores, se promueve que haya un trabajo de estas cuestiones en particular en una subasignatura dentro de la asignatura de Lengua Castellana, que es la que recibe el nombre de "Lengua +1 ".

En cuanto a los porcentajes, la evaluación de la asignatura de Lengua Castellana en este curso queda dividida tal y como se refleja en la Figura 1. En estos porcentajes se incluyen los resultados obtenidos en el +1 . Finalmente, cabe añadir que esta evaluación es continua por lo que el estudiante puede ir, progresivamente, desarrollando sus competencias y alcanzar los objetivos a lo largo del curso.

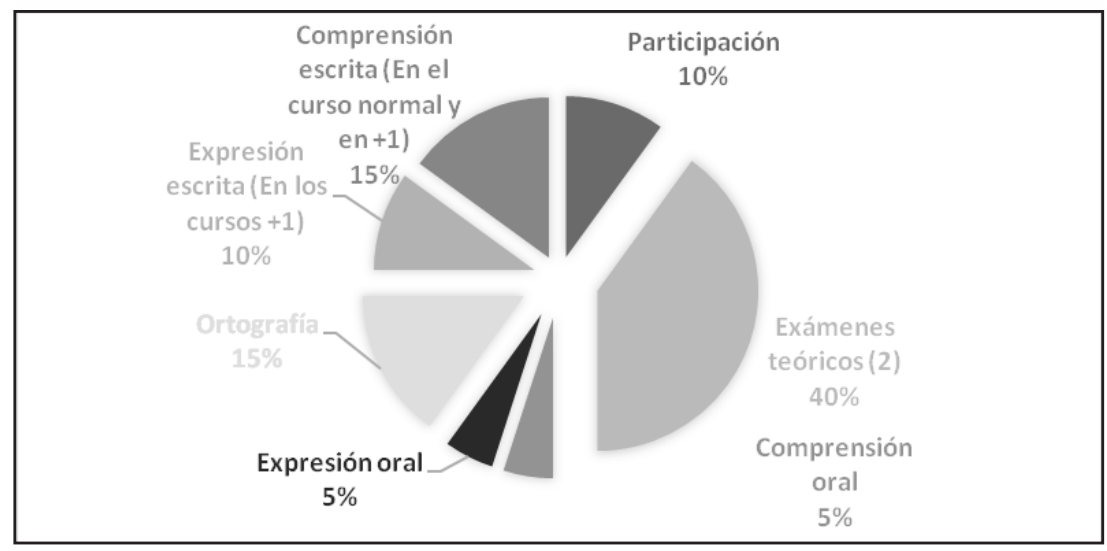

Figura 1: Sistema de evaluación de $1^{\circ} \mathrm{ESO}$ 
En el programa de esta materia también se concreta en la metodología empleada en el aula. Por lo general, se presta especial atención a cuáles van a ser las técnicas de aprendizaje cooperativo que se van a utilizar y en qué contextos se optará por una u otra técnica. Asimismo, se ofrecen directrices acerca de las intervenciones que va a llevar a cabo el docente de acuerdo a la recuperación de los conocimientos previos, el correcto aprovechamiento de la asignatura y la gestión del tiempo. De la misma manera, se incide en la importancia de presentar los nuevos contenidos no de manera aislada, sino interrelacionándolos con lo que los estudiantes ya han estudiado. Se considera que, de ese modo, podrán ir construyendo sus nociones y estableciendo relaciones que faciliten el aprendizaje.

Es también en este apartado de metodología donde se especifica el sistema de trabajo que se llevará a cabo en la ya mencionada subasignatura denominada Lengua +1 . Esta sección, todavía en fase de pilotaje y diseño, es impartida por un profesor diferente al que imparte la asignatura de Lengua Castellana y Literatura. Al igual que en el programa general, también se especifican las técnicas de trabajo cooperativo que se van a emplear (1-2-4, folio giratorio, lápices al centro, lectura compartida...) y se desglosan los tipos de metodología que van a aplicar los docentes.

Destaca la importancia asignada a la génesis de un diario de aprendizaje en el que recopilar los diferentes aprendizajes que se van adquiriendo y el objetivo de trabajar la autoevaluación y la revisión conjunta con el fin de indagar en las dificultades que cada estudiante tiene en el manejo de estas dos destrezas. Un análisis más detallado del programa específico de esta subasignatura deja comprobar que cada evaluación se construye en torno a la distinción entre textos discontinuos y continuos. Dentro de la categoría de continuos se encuentran las diferentes tipologías que se van a tener en cuenta. Así, el curso quedará dividido tal y como se indica en la Tabla 2.

\begin{tabular}{lll}
\hline Trimestre & Comprensión escrita & Expresión escrita \\
\hline 1er trimestre & $\begin{array}{l}\text { resúmenes, exposiciones divul- } \\
\text { gativas, textos instructivos, no- } \\
\text { ticias. }\end{array}$ & $\begin{array}{l}\text { entrevista, correos electrónicos, car- } \\
\text { tas y ortografía (ortografía de las le- } \\
\text { tras). }\end{array}$ \\
\hline $\mathbf{2}^{\mathbf{0}}$ trimestre & $\begin{array}{l}\text { textos instructivos, expositivos y } \\
\text { comics. }\end{array}$ & $\begin{array}{l}\text { noticias, textos instructivos y expo- } \\
\text { sitivos, cartas, correos electrónicos y } \\
\text { ortografía (acentuación). }\end{array}$ \\
\hline 3er trimestre & $\begin{array}{l}\text { textos poéticos, descriptivos y y } \\
\text { narrativos, fábulas, y otras figu- } \\
\text { ras retóricas. }\end{array}$ & $\begin{array}{l}\text { textos narrativos, descriptivos y poé- } \\
\text { ticos diálogos y ortografía (puntua- } \\
\text { ción) }\end{array}$ \\
\hline
\end{tabular}

Tabla 2: Configuración piloto del curso Lengua +1

\section{Resultados y propuesta}

A partir de la revisión de la documentación oficial, así como de los principios del enfoque basado en los géneros, se presenta, a continuación, una propuesta didáctica para el marco de trabajo de la previamente mencionada subasignatura Lengua +1 . A pesar de que la propuesta que aquí se presenta está orientada al castellano como ocu- 
rre en el centro seleccionado, se trata de una propuesta que se podría aplicar también para la enseñanza y refuerzo de la lengua vasca, sin olvidar la transferibilidad de los conocimientos adquiridos.

\subsection{Aspectos generales de la propuesta}

La subasignatura Lengua +1 está programada para impartirse durante una de las sesiones semanales de la asignatura de Lengua. El curso estaría dividido en tres trimestres, dividido, a su vez, en 10-11 semanas cada uno de ellos. Esta propuesta se construye sobre el trabajo con seis géneros, por lo que cada uno de ellos se trabajaría a lo largo de cinco sesiones y quedaría entre ellos una sesión de eslabón o transición para la recuperación de conocimientos.

Tal y como se observa en la Figura 2, el resultado de esta programación daría lugar a un curso dividido en seis tipos de textos que corresponderían a seis géneros discursivos distintos, seleccionados de acuerdo a la clasificación de Sánchez (2006). Es importante incidir en que la distribución y la organización no se ha realizado de manera aleatoria, sino que se ha buscado, de acuerdo a las directrices de este enfoque, que exista un hilo conductor que permita avanzar en el desarrollo de esta competencia mientras se hace uso de los conocimientos previos. Asimismo, cabe añadir que, en la medida de lo posible, se espera que los géneros se trabajen a través de canales virtuales.

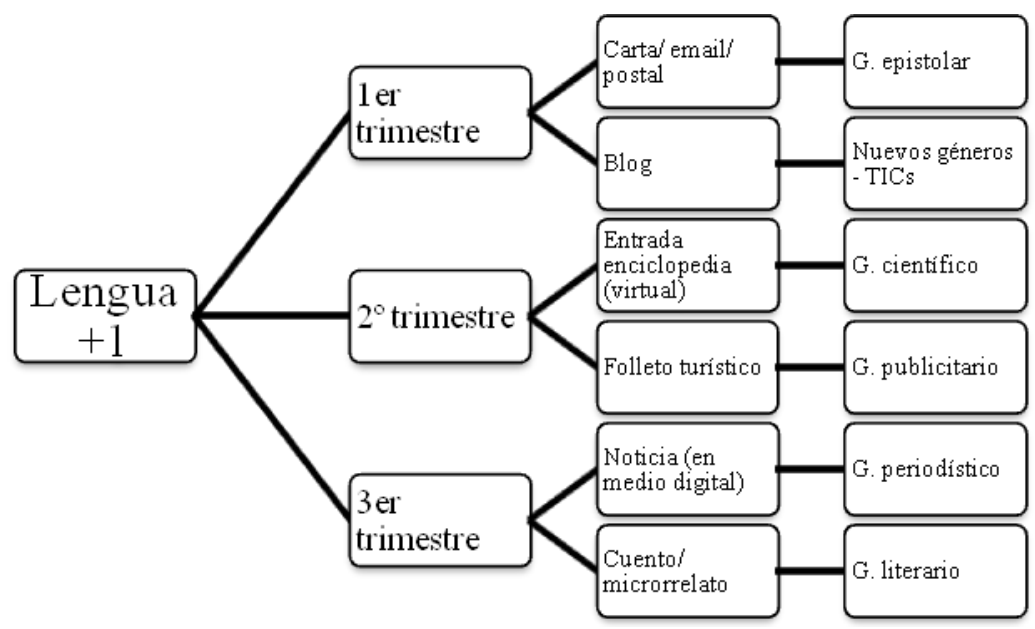

Figura 2: Organización global de la propuesta didáctica.

En este trabajo, el objetivo es ofrecer la propuesta correspondiente al primer género del primer cuatrimestre como punto de partida del modelo de trabajo y del nivel esperado. No obstante, cabe recordar que en este enfoque se entiende que el estudiante va engranando su nuevo conocimiento sobre el conocimiento anterior. Por ello, se esperaría que en el trabajo de los sucesivos géneros se hiciese uso del conocimiento anterior de manera estratégica. Además, según avanzase el curso, el docente tendría que ir progresivamente distanciándose del estudiante para que éste pueda desarrollar su autonomía y pueda ir adoptando una actitud crítica con respecto al proceso de aprendizaje 
en el que está inmerso. Por todos estos motivos, el diseño de las unidades didácticas de los otros géneros no sería idéntico al que aquí se presenta.

Es importante mencionar, también, el modo en que se evaluará la efectividad de este sistema de enseñanza. Se considera esencial que, tanto a lo largo del curso, como al final, se adopte una actitud crítica con respecto a la aplicación de este enfoque. Para ello, se proponen los siguientes sistemas de evaluación: (a) contraste de las producciones iniciales de los estudiantes y de las producciones finales, (b) cuestionarios de valoración a los estudiantes y profesores participantes y (c) reuniones periódicas de los docentes que imparten esta materia para realizar grupos focales que permitan diagnosticar los puntos fuertes y débiles de la enseñanza que se está llevando a cabo.

\subsection{Ficha técnica, competencias y objetivos didácticos}

Área implicada: Lengua Castellana y Literatura

Formación en la que se enmarca: Desarrollo de la competencia escritora

Nivel: $1^{\circ}$ de la ESO - primer trimestre, ler período

$\mathrm{N}^{\mathrm{o}}$ de sesiones: 5 sesiones

Duración de las sesiones: 55 minutos (tiempo semanal establecido por el centro para el curso de Lengua +1 ).

Tabla 3: Ficha técnica

Las competencias básicas trabajadas ${ }^{3}$ son las siguientes:

- Comunicación lingüística: en todas las actividades se trabajará la comunicación lingüística en tanto que se tienen en consideración todos los componentes que conforman esta competencia: el lingüístico, el pragmático-discursivo, el sociocultural, el estratégico y el personal.

- Tratamiento de la información y competencia digital: tareas 11 y 12.

- Aprender a aprender: en todas las tareas subyace el objetivo de que el estudiante desarrolle esta competencia y, por extensión, tenga lugar un control de los propios procesos de aprendizaje que se ajuste a las demandas de cada tarea. El objetivo último es que el estudiante sea cada vez más autónomo y su aprendizaje sea más eficaz.

- Social y ciudadana: casi la totalidad de las actividades de este taller se llevan a cabo con una metodología de trabajo colaborativo cuyo objetivo es que el estudiante adquiera las destrezas necesarias para ser parte de esta sociedad y actuar en ella de manera cívica, asumiendo los valores de la misma como propios y desarrollando estrategias para alcanzar una comunicación eficaz con los demás miembros de la misma.

- Conciencia cultural y artística: tareas 10, 11, 12 y 13.

- Autonomía e iniciativa personal: todas las tareas realizadas a lo largo de estas sesiones tienen como principio el desarrollo de esta competencia a diferentes niveles: capacidad de análisis, capacidad para la planificación, gestión...,

Las competencias aquí presentadas se han ajustado a las directrices de la LOE que es la ley que rige la educación en el centro educativo seleccionado en el momento del diseño de esta propuesta. No obstante, se ha comprobado que éstas podrían ser trasladables a las directrices de la LOMCE una vez esta ley estuviera en vigor. 
toma de decisiones, negociación, liderazgo, pensamiento crítico, evaluación, autoevaluación, gestión del tiempo...

Los objetivos didácticos generales de esta propuesta de intervención didáctica son los siguientes:

- Desarrollar las habilidades lingüísticas de manera colaborativa.

- Recuperar de manera automática los conocimientos previos y relacionarlos con los nuevos conocimientos.

- Cooperar para dar respuesta óptima a situaciones reales de comunicación.

- Adoptar una actitud crítica con respecto a la propia producción escrita.

- Emplear recursos digitales para la mejora del conocimiento metalingüístico.

- Realizar una interpretación crítica de textos del género epistolar.

- Reconocer los rasgos de los textos propios del género epistolar, así como las características lingüísticas.

- Integrar los conocimientos metalingüísticos a los usos significativos de la lengua.

Estos podrán manifestarse en una o varias sesiones a lo largo de la secuencia didáctica.

\subsection{Descripción de la actividad}

A continuación, se presenta una tabla (ver Tabla 4) en la que se desglosan las actividades propuestas.

\begin{tabular}{|l|l|}
\hline SESIÓN & DESARROLLO \\
\hline 1 & $\begin{array}{r}\text { Elicitación de saberes previos acerca del género epistolar. Contex- } \\
\text { tualización según la realidad del estudiante. Lluvia de ideas en gru- } \\
\text { po y puesta en común en toda la clase. }\end{array}$ \\
2. & $\begin{array}{l}\text { Dinámicas grupales para reconocer las características de la carta: el } \\
\text { sobre y el texto. } \\
\text { a. } \quad \text { Características del sobre: léxico (anverso, reverso, remi- } \\
\text { te...) }\end{array}$ \\
3. $\begin{array}{l}\text { Distinción entre carta formal e informal. En grupo pequeño y puesta } \\
\text { en común con toda la clase. Identificación de cuándo y por qué se } \\
\text { escribe una carta formal o informal. Elicitación de experiencias pre- } \\
\text { vias. Introducción del concepto de registro. } \\
\text { a. } \quad \begin{array}{l}\text { Partes constitutivas de las cartas formales e informales: di- } \\
\text { ferencias y similitudes. }\end{array} \\
\text { b. Saludos y despedidas: recursos lingǘsticos }\end{array}$ \\
$\begin{array}{l}\text { Estas actividades se irán escribiendo en un libreto facilitado por el docente } \\
\text { para que tengan un lugar en el que ir reflejando los nuevos conocimientos } \\
\text { adquiridos. }\end{array}$ \\
\hline
\end{tabular}




\begin{tabular}{|c|c|}
\hline 2 & $\begin{array}{l}\text { 4. Recuperación de los conocimientos adquiridos en la sesión anterior. } \\
\text { Reflexión sobre la utilidad de dichos conocimientos. } \\
\text { Tarea de escritura 1: la carta formal. "Estamos en campaña electo- } \\
\text { ral y una futura alcaldesa pedido a los ciudadanos sugerencias para } \\
\text { mejorar los barrios. Escribe una carta y cuéntale qué problemas ob- } \\
\text { servas en tu zona"'. } \\
\text { a. Actividades de planificación del texto: } \\
\text { i. Trabajo con la situación e intención comunicati- } \\
\text { vas } \\
\text { ii. Grupalmente planear la estructura y organización } \\
\text { del contenido. } \\
\text { b. Recursos para la escritura: se ofrecen conectores y se expli- } \\
\text { c. Textualización individual de la carta } \\
\text { d. Textualización de la información del sobre }\end{array}$ \\
\hline 3 & $\begin{array}{l}\text { 6. Recuperación de los conocimientos adquiridos en la sesión anterior. } \\
\text { Reflexión grupal sobre la utilidad de dichos conocimientos. } \\
\text { 7. Revisión del texto escrito de manera cooperativa. Técnica del folio } \\
\text { giratorio, cronometrado y en cinco tiempos. Niveles de revisión: es- } \\
\text { tructura y organización, contenido, léxico, gramática y el sobre. Se } \\
\text { pone en común, previamente, qué se puede corregir en cada caso. } \\
\text { a. Presentación de aspectos metalingüísticos para una mejor } \\
\text { revisión de los textos. Orientaciones. } \\
\text { 8. Entrega de la carta (sobre y texto) al docente. }\end{array}$ \\
\hline 4 & $\begin{array}{l}\text { 9. Síntesis de los puntos fuertes y de las debilidades diagnosticadas por } \\
\text { el docente en la revisión de las cartas. Se destacan los beneficios de } \\
\text { la planificación y la revisión grupal. } \\
\text { 10. Contextualización de la nueva tarea de lectura. Se recupera la idea } \\
\text { de que la carta y el email no es lo mismo. Se presenta el tema: el } \\
\text { conflicto de Siria y los refugiados. } \\
\text { 11. Búsqueda grupal de información en medios virtuales acerca de la } \\
\text { localización de Siria. } \\
\text { 12. Trabajo en valores: el concepto de refugiado. Visualización de ví- } \\
\text { deo explicativo sobre la diferencia entre inmigrante y refugiado. Re- } \\
\text { flexión. Importancia de la tolerancia, la solidaridad... } \\
\text { 13. Lectura individualizada de un email de ACNUR y preguntas acerca } \\
\text { de contenido, los elementos paratextuales... de manera grupal. } \\
\text { 14. Reconocimiento de las partes constitutivas del correo electrónico. } \\
\text { Contraste con lo aprendido en relación a la carta. Trabajo con la } \\
\text { terminología: spam, CC, adjunto, descartar... Elicitación de saberes } \\
\text { previos. }\end{array}$ \\
\hline
\end{tabular}

Las propuestas temáticas están sujetas a modificaciones según los contextos en los que se aplique esta propuesta. 


\begin{tabular}{|l|l|}
\hline 15. Recuperación de los conocimientos adquiridos en la sesión anterior. \\
Reflexión acerca de la utilidad de dichos conocimientos. \\
16. Tarea de escritura 2: el correo electrónico "El centro en el que estu- \\
dias va a acoger durante dos semanas a unos estudiantes refugiados. \\
Preséntate, dales la bienvenida y cuéntales información útil acerca \\
de la vida en tu ciudad". \\
a. Actividades de planificación: reconocimiento de la situa- \\
ción comunicativa e identificación del canal (característi- \\
cas, particularidades, etc.). \\
b. Planificación del contenido del texto de manera grupal. \\
c. Textualización de manera grupal \\
i. Envío de un correo electrónico al docente.
\end{tabular}

Tabla 4: Descripción de las actividades propuestas para el trabajo del género epistolar por sesiones

Las mesas estarán dispuestas en grupos de 4 ó 5 y habrá espacios entre grupo y grupo para que el docente pueda moverse sin dificultad. El proyector se encontraría en la parte frontal para ser visible para todos los estudiantes.

Para una ejecución adecuada de la secuencia didáctica será necesario disponer de los siguientes recursos en el aula:

- Ordenador y proyector.

- Un dispositivo (móvil, Tablet...) con conexión a Internet por grupo para llevar a cabo las actividades que requieren de acceso a la red.

- No será indispensable disponer de una pizarra digital, pero, en el caso de disponer de ella, se empleará para, a partir de los mismos materiales, generar otro tipo de dinámicas en el aula.

- Libretos de hojas.

Esta secuencia puede llevarse a cabo, de manera adaptada, a un entorno en el que no se tenga acceso a las nuevas tecnologías o en los que pueda haber dificultades debido a la brecha digital.

\subsection{Evaluación}

Los indicadores que se emplearán serán los siguientes:

El estudiante es capaz de:

- Trabajar de manera colaborativa y contribuir a un entorno adecuado de trabajo por equipos en el que existe una interdependencia positiva entre los miembros.

- Emplear los conocimientos previos de manera estratégica para interiorizar y emplear los nuevos.

- Identificar las características de situaciones reales de comunicación para dar respuesta óptima a las demandas comunicativas.

- Adoptar una actitud crítica con respecto a la propia producción escrita. 
- Emplear diversos recursos tecnológicos para una mejor consecución de las tareas.

- Reconocer los rasgos propios de los textos del género epistolar a nivel discursivo, pragmático y lingüístico.

En cuanto a las herramientas y técnicas para la evaluación de la escritura se propone que haya un seguimiento tanto de los procesos que ocurren antes de empezar a escribir, como de los que tienen lugar durante y después de la escritura.

\section{Conclusiones}

En este trabajo se ha llevado a cabo una revisión de cuál es la presencia que tiene la escritura en las directrices oficiales, cuál es la presencia que esta habilidad tiene en las aulas y desde qué enfoques se aborda su proceso de enseñanza-aprendizaje. Entre los resultados más reseñables de esta revisión teórica, se encuentra la primacía del concepto de $\mathrm{CC}$ y la estructuración de todas las enseñanzas asociadas a la lengua en torno a este concepto. Se trata éste de un enfoque que rompe con los modos de enseñanza tradicional y que busca la creación de hablantes competentes dentro de los diferentes contextos discursivos que tienen lugar en las lenguas que emplea. Así también, en este trabajo se ha indagado en el género discursivo como eje para la programación de un curso de escritura en la etapa educativa de secundaria.

No obstante, la aportación más significativa de este trabajo es el diseño de una propuesta didáctica que aúna las directrices oficiales, los objetivos didácticos del centro, una metodología colaborativa e integradora y, por último, una enseñanza que aborda la escritura junto con conocimientos de tipo discursivo, pragmático y lingüístico. No hay que olvidar que es, a su vez, una propuesta que, pese a que se haya diseñado para este centro en particular, podría ser extrapolable a otros contextos educativos y a otras lenguas.

Este estudio permite, además, establecer un marco de trabajo para futuras investigaciones y líneas de trabajo asociadas a la enseñanza de la escritura en secundaria; campo que, por otra parte, se ha demostrado no estar suficientemente trabajado. En este sentido, resultaría de interés llevar a cabo una investigación en acción que permitiese comprobar qué tipo de viabilidad tiene esta propuesta y observar qué tipo de resultados se obtienen en los estudiantes. La creación de un grupo de control y uno experimental ayudaría a efectuar mejoras en la propuesta que aquí se ha presentado y seguir avanzando en innovación educativa en el ámbito de la lengua. De la misma manera, sería de interés avanzar en el diseño del curso completo asociado a la subasignatura Lengua +1 para observar cómo sería el trabajo de los diferentes géneros.

\section{Bibliografía}

Adam, J. (1992): Les textes: types et prototypes. Recit, description, argumentation, explication et dialogue, París, Nathan.

Alexopoulou, A. (2010): "El enfoque basado en los géneros textuales y la evaluación de la comptencia discursiva", en Actas del XXI Congreso Internacional de ASELE (97-110). Salamanca, Universidad de Salamanca. 
Álvarez, M. y Yañiz, C. (2015): "Las prácticas escritas en la universidad española", Cultura y Educación, 27(3), 594-628.

Arias, D. (2013): "La escritura como proceso, como producto y como objetivo didáctico. Tareas pendientes", en Actas del CEDELEQ IV (33-46). Montreal, Universidad de Montreal.

Bajtin, M. (1979): “El problema de los géneros discursivos”, Estética de la creación verbal, México, Siglo X.

Björk, L. y Blomstrand, I. (2006): La escritura en la enseñanza secundaria. Los procesos del pensar y del escribir, Barcelona, Graò.

Camps, A. (2003). Secuencias didácticas para aprender a escribir, Barcelona, Graò.

Camps, A. y Castelló, M. (2013): "La escritura académica en la universidad", Revista de Docencia Universitaria, 11(1), 17-36.

Carlino, P. (2005): Escribir, leer y aprender en la universidad. Una introducción a la alfabetización académica, Buenos Aires, Fondo de Cultura Económica.

Castelló, M. (2006): "La escritura epistémica: enseñar a gestionar y regular el proceso de composición escrita”, en Actas del Congreso Internacional de Educación, Investigación y Formación Docente (229-243), Medellín, Universidad de Antioquia.

Chaisiri, T. (2010): "Implementing a Genre Pedagogy to the Teaching of Writing in a University Context in Thailand", Language Education in Asia, 1, 181-199.

Consejo de Europa (2001): Marco Común Europeo de Referencia para las Lenguas: aprendizaje, enseñanza, evaluación, Madrid, Instituto Cervantes-Ministerio de Educación Cultura y Deporte, Anaya, 2002.

Eurydice (2002): Competencias clave. Un concepto en expansión dentro de la educación general obligatoria. http: //www.eurydice.org [03.06.2016].

Feez, S. (1998): Text-Based Syllabus Design, Sydney, McQuarie University/AMES.

Gobierno Vasco (2010): Decretos curriculares para la Educación Infantil, Básica y Bachillerato de la Comunidad Autónoma del País Vasco, Vitoria-Gasteiz.

Gobierno Vasco (2011): Orientaciones didácticas para la materia Lengua Castellana y Literatura, Vitoria-Gasteiz.

Gobierno Vasco (2014): Heziberri 2020. Marco del modelo educativo pedagógico, Vitoria-Gasteiz.

González-Caravaca, G. (2014): “Tareas de escritura y géneros discursivos en los libros de texto", Bellaterra Journal of Teaching \& Learning Language \& Literature, 7 (2), 70-83.

Hernández, A. y Quintero, A. (2001): Comprensión y composición escrita. Estrategias de aprendizaje, Madrid, Síntesis.

Hyland, K. (2003): “Genre-Based Pedagogies: A Social Response to Process", Journal of Second Language Writing, 12, 17-29.

Hyland, K. (2004): Genre and Second Language Writing, Ann Arbor, MI, University of Michigan Press.

Hyland, K. (2007): “Genre pedagogy: Language, literacy and L2 writing instruction”, Journal of Second Language Writing, 16, 148-164.

Hymes, D. H. (1971): “Acerca de la competencia comunicativa”, en Llobera et al. (1995). Competencia comunicativa. Documentos básicos en la enseñanza de lenguas extranjeras, Madrid, Edelsa, 27-47.

Instituto Cervantes (2007): Plan Curricular del Instituto Cervantes. Niveles de referencia para el español, Madrid, Biblioteca Nueva.

Instituto Cervantes Diccionario ELE. Recuperado de http: //cvc.cervantes.es/ensenanza/biblioteca_ele/diccio_ele/diccionario/competenciacomunicativa.htm[10.12.2016]. 
Martin, J. R. (2001): “Cohesion and Texture”, en Schiffrin, D., Tamen, D. y Hamilton, H. E., The Handbook of Discourse Analysis (35-53), Massachusetts, Blackwell Publishing.

Matsuda, P. K., Canagarajah, A. S., Harklau, L., Hyland, K. y Warschauer, M. (2003): "Changing Currents in Second Language Writing Research: A Colloquium", Journal of Second Language Writing, 12,151-179.

Ministerio de Educación y Ciencia. (2013): Ley orgánica para la mejora de la calidad educativa (LOMCE) (Ley Orgánica 8/2013, 9 de diciembre), en Boletín Oficial del Estado.

Moyano, E. (2005): "Una propuesta para la enseñanza de la lectura y la escritura basada en la teória de género y registro de la LSF: resultados de una investigación e implementación en diferentes niveles educativos", II Conferencia Latinoamericana de Lingüística Sistémico-Funcional (1-15), Concepción, Chile, Universidad Católica de la Santísima Concepción.

Parodi, G. (2008): “Géneros discursivos y lengua escrita: propuesta de una concepción integral desde una perspectiva sociocognitiva”, Letras, 51(80),19-55.

Raimes, A. (1998): “Teaching writing”, Annual Review of Applied Linguistics, 18, 142-167.

Sánchez, J. (2006): Saber escribir, Madrid, Aguilar/Instituto Cervantes.

Serrano, S. (2014): "La lectura, la escritura y el pensamiento. Función epistémica e implicaciones pedagógicas", Lenguaje,17(1), 97-122.

Swales, J. (1996): Genre Analysis. English in Academic and Research Settings, Cambridge, Cambridge University Press.

Werlich, E. (1975): Typologie der Texte, Munich, Fink.

Zayas, F. (2012): "Los géneros discursivos y la enseñanza de la composición escrita", Revista Iberoamericana de Educación, 59, 63-85.

Zayas, F. y Pérez Esteve, P. (2012): Los géneros discursivos como eje de los proyectos lingüisticos de centro. Recuperado de http://goo.gl/qRh9R[25.11.2016]. 